\title{
Spectral broadening effects of high-power amplifiers in MIMO-OFDM relaying channels
}

\author{
Ishtiaq Ahmad ${ }^{1}$, Ahmed lyanda Sulyman ${ }^{1 *}$, Abdulhameed Alsanie' ${ }^{1}$, Awad Kh Alasmari ${ }^{1}$ and Saleh A Alshebeili ${ }^{1,2}$
}

\begin{abstract}
The combination of MIMO-OFDM is a very attractive solution for broadband wireless services. Thus, the two prominent fourth-generation (4G) cellular systems, WiMAX and LTE-advanced, have both adopted MIMO-OFDM transmission at the physical layer. OFDM signal however suffers from nonlinear distortions when passed through high-power amplifier (HPA) at the RF stage. This nonlinear distortion introduces out-of-band spectral broadening and in-band distortions on the transmitted signals. $4 \mathrm{G}$ cellular standards have placed strict limits on the allowable spectral broadening in their spectrum mask specifications, to insure that data transmission on a given channel is not interfering significantly with an adjacent channel user. In this article, we characterize the out-of-band spectral broadening introduced by HPA when MIMO-OFDM signals are transmitted over multiple relaying channels.

Expressions for the power spectral density of MIMO-OFDM signals are derived over multiple relay channels, and the cumulative effects of HPA on the spectrum of the transmitted signals are estimated. It is shown that depending on the number of relays and the relaying configuration employed, it may happen that a transmitted MIMO-OFDM signal with the transmit spectrum mask initially within the allowable set limit at the source node arrives at the destination violating this limit due to the cumulative effects of the multiple HPA's in a multihop relaying channel.
\end{abstract}

Keywords: Spectral re-growth, Amplifier nonlinearity, Spectral mask, MIMO-OFDM, Relaying channels

\section{Introduction}

Fourth-generation (4G) broadband communication systems need to provide ultra-high data rate services in order to meet the requirements of future highbandwidth multimedia applications over cellular systems such as the digital TV distributions and interactive videos planned in the WiMAX and LTE-advanced. Among the candidate physical layer technologies that can be deployed to achieve these goals, MIMO-OFDM is the most potent solution that can provide such high data rate at high spectral efficiencies. Compared to the single-carrier systems however, OFDM has a large peakto-average-power ratio $[1,2]$ which makes it very sensitive to high-power amplifier (HPA) nonlinearities at the RF stage of the transmission chain [3]. There are two important effects of the HPA nonlinearities introduced in the transmitted OFDM signals: in-band and out-ofband distortions. The in-band distortion degrades bit

\footnotetext{
* Correspondence: asulyman@ksu.edu.sa

${ }^{1}$ Department of Electrical Engineering, King Saud University, Riyadh, Saudi Arabia

Full list of author information is available at the end of the article
}

error rate (BER) performance and capacity of the cellular operator [4-10], whereas the out-of-band distortion arising from the spectral broadening effect of the HPA affects other users operating in the adjacent frequency bands [11-15]. While the BER and capacity analysis of OFDM relay links in the presence of HPA nonlinearity are fairly well understood [5 and references there in], the effects of HPA on out-of-band emissions for OFDM relay link are not yet studied to the best of the authors' knowledge. Out-of-band emissions are strictly monitored by the cellular regulators using the concept of transmit spectrum mask. Transmit spectrum mask is the power contained in a specified frequency bandwidth at certain offsets, relative to the total carrier power. In the $4 \mathrm{G}$ system such as WiMAX and LTE-advanced, strict limits have been specified for the spectrum masks. Figure 1 displays the spectrum mask of the IEEE 802.16 signal specified in the WiMAX standard [16], where about $25-\mathrm{dB}$ attenuation is required between the reference carrier power and all unwanted spurious emissions at certain frequency offsets from the operating bandwidth.

\section{Springer}




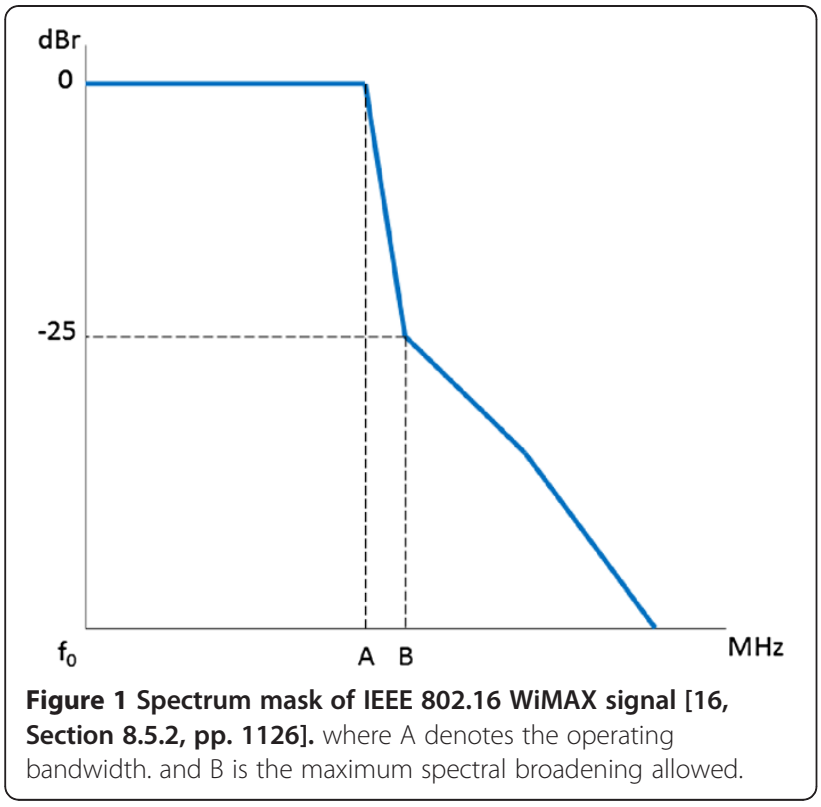

Banelli and Cacopardi [11] derived analytical expressions for the correlation function of the output of nonlinear HPA when the input to the amplifier is an OFDM signal. The power spectral density (PSD) of the signal is then calculated using the Fourier transform of the correlation function. Grad et al. [12] studied spectral re-growth due to HPA nonlinearity in code-division multiple access (CDMA) systems. They obtained analytical expressions for the power spectrum of the CDMA signal at the output of the HPA, using a complex power-series model for the HPA characteristics. The out-of-band emission for the time division synchronous CDMA system is presented in [13], in terms of third-order intercept point (IP3). Cottais et al. [14] derived expressions for the PSD of a general multicarrier signal at the output of a memoryless HPA. They also obtained a closed-form expression for the PSD of the special case of single-carrier signals. Helaly et al. [15] examined the effects of the characteristics of the input CDMA signal on the resulting out-of-band spectral regrowth at the output of the HPA. They pointed out that, in addition to the HPA saturation level, the input signal's threshold crossing rate and the variance of the clipped signal also contribute to the spectral re-growth. It is important to note that OFDM signals share some similarities with CDMA signals in this regard. Recently also, Gregorio et al. [17] proposed a MIMO-predistortion (MIMO-PD) system that tries to compensate crosstalk and IQ imbalance in single-hop MIMO-OFDM communication systems, where they have shown that some reduction in the spectral regrowth can be achieved using the proposed MIMO-PD system. The effectiveness of such a compensation scheme in a multihop environment is however not yet known.
All the above-cited studies, and several others in the literature however, focused on the spectral re-growth due to HPA nonlinearity in a single-hop communication system. Recently, the two prominent 4G cellular systems, WiMAX and LTE-advanced, have defined relaying as an integral part of the network design $[18,19]$. Thus, MIMO-OFDM signals transmitted in the $4 \mathrm{G}$ systems will frequently pass through one or more relay hops from source node to the destination node. Investigating the level of adherence to set limits on spectral broadening in cellular systems employing relaying technologies is therefore a deployment imperative. To the best of the authors' knowledge, no work has presented a detailed study of the broadening effects of HPA nonlinearity on the spectrum of MIMO-OFDM signals in multihop relaying channels.

In this article, we characterize for the first time in the literature, the cumulative spectral broadening effects of multiple HPAs when MIMO-OFDM signals are transmitted over multihop relaying channels. Expressions for the PSD of a MIMO-OFDM signal are presented over multihop relay channels, each equipped with nonlinear HPA's. It is shown that due to the cumulative effect of the multiple HPA's in a MIMO link, and the cascade effect of many relaying channels in a multihop relay link, significant broadening effect occur which is much more than what would be observed in a single-hop transmission such as those characterized in [11-15]. We also show that for the amplify-and-forward (AF) and demodulate-and-forward (DemF) relaying options, the resulting cumulative re-growth may lead to spectral mask violations after a few relaying hops is traversed by the transmitted OFDM signal, even though the set limits were initially met at the source node [at the base station (BS) for downlink transmission, or at the mobile station (MS) for uplink transmission]. For the decode-and-forward (DF) relaying option, it is observed that less severe spectral broadening are observed. However, due to the latency problems associated with the DF relaying option which degrades quality of broadband signals, AF or DemF are the preferred candidates for broadband transmissions over relaying channels and therefore the spectral broadening issues observed here must be given considerable attentions in the design of broadband multihop relaying systems.

\section{HPA nonlinearity model for MIMO-OFDM relaying channel}

Consider a MIMO-OFDM relaying system employing $n$ subcarriers per OFDM symbol, $M$ transmitting and $L$ receiving antennas at each hop in the transmission chain. We assume that all the transmitting antennas at each node simultaneously transmit different symbols (MIMOmultiplexing system), and that all $L$ receiving antennas 
at each receiving node are expended in separating each of the $M$ transmitted streams. For simplicity, we consider the case of $N$ transmitting and $N$ receiving antennas (where $N \leq M, L$ ). Thus, in the ensuing analysis we focus on the $N \times N$ MIMO-OFDM multiplexing relaying system. We consider that the transmitted signal from a source node passes through a single-hop MIMO channel $H_{0}$, and $R$ multihop MIMO relaying channels $H_{1}, \ldots, H_{R}$, associated with $R$ fixed or mobile relaying nodes, to the destination node as shown in Figure 2.

The MIMO channel matrix for each $i$ th hop transmission $\mathrm{H}_{i}, i=0,1, \ldots, R$, is an $n N \times n N$ block diagonal matrix, with the $k$ th block diagonal entries $H[K]_{i}$ corresponding to the fading on the $k$ th OFDM subcarrier, $k=0,1, \ldots, n-1$, modeled as independent and identically distributed (iid) random variables taken from zero mean complex Gaussian distribution, with unit variance. We assume that the set of random matrices $\left\{H_{0}, \ldots, H_{R}\right\}$ are independent, and that AF, DemF, or DF relaying options could be employed at the relay nodes $[5,20]$. We also assume that the $\mathrm{BS}$ and all the relay stations (RS's) employ similar nonlinear HPA's which introduce similar nonlinear distortions per hop, in the transmitted signal. A consequence of this assumption is that if the BS or any of the RS employ a nonlinear HPA with nonlinearity level more or less than the other devices, then the contribution of that device to the overall spectral broadening will be more or less than estimated in this analysis. We can express the transmitted symbol in polar coordinate as

$$
x(t)=r(t) e^{j \theta(t)}
$$

where $r(t)$ is the amplitude and $\theta(t)$ is the phase of the input signal into the HPA. The signal at the output of the HPA can then be expressed as

$$
\hat{x}(t)=g[r(t)] e^{j \theta(t)}
$$

where $g[r(t)]$ is a complex nonlinear distortion function, which only depends on the envelope of the transmitted symbols. The nonlinear distortion function can be expressed as

$$
g[r(t)]=g_{A}[r(t)] e^{j g_{P}[r(t)]}
$$

where $g_{A}[r(t)]$ is the amplitude-to-amplitude (AM-AM) and $g_{P}[r(t)]$ is the amplitude-to-phase (AM-PM) conversions of the HPA. The AM-AM conversions for different memoryless HPA models [Saleh model, SolidState Power Amplifier (SSPA) model, and soft envelop limiter (SEL) model] used in communication systems are plotted in Figure 3.

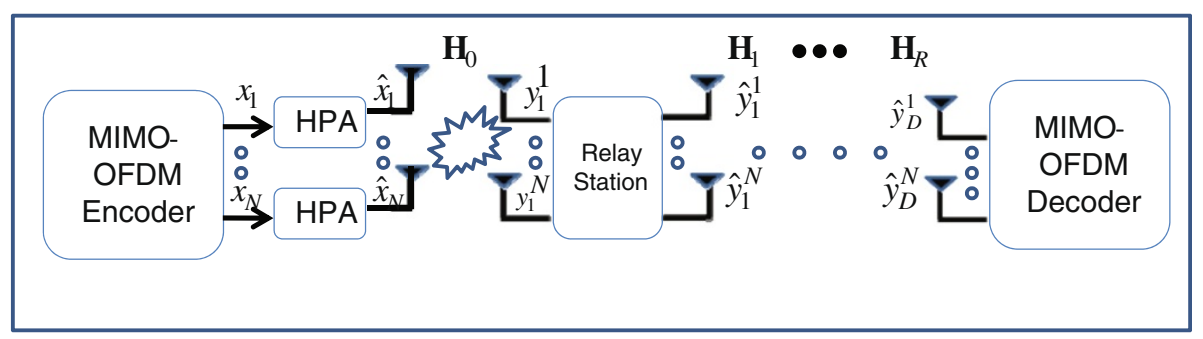

(a)Nonlinear Multi-Hop MIMO-OFDM Communication Channel

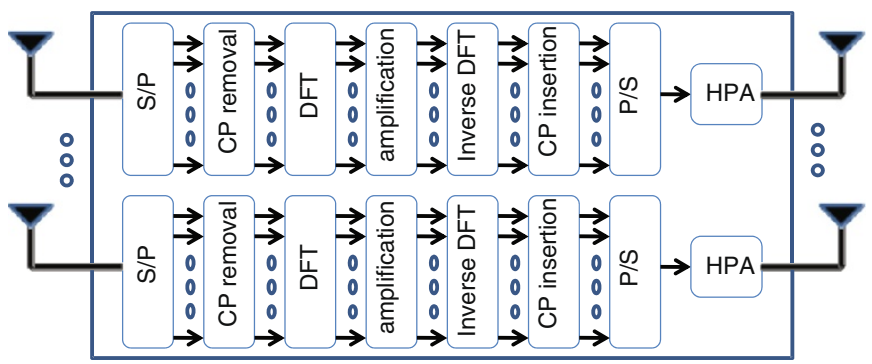

(b) Signal Processing at Relay Station (RS)

Figure 2 HPA nonlinearity model for MIMO-OFDM relaying channel. 


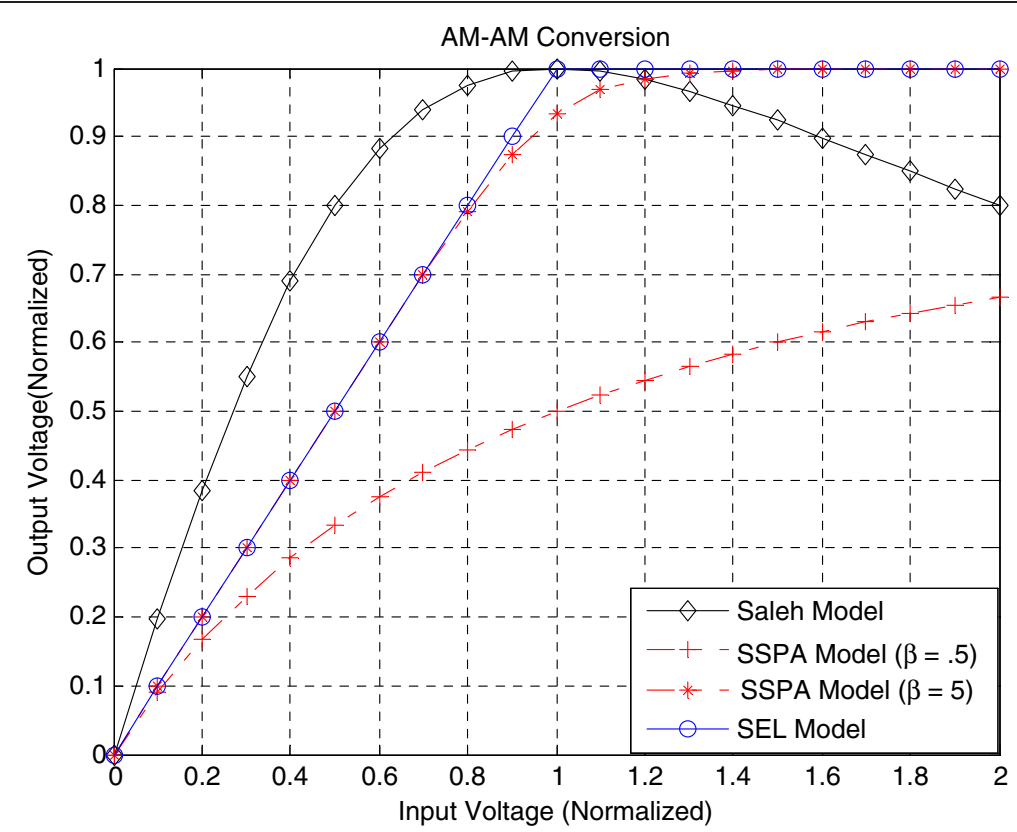

Figure 3 AM-AM conversions for different amplifier models.

It is easily observed from this figure that Saleh model depicts the severest nonlinear distortions followed by the SSPA model. The SEL model on the other hand depicts more closely the current state-of-the-art in HPA designs for cellular systems where approximately linear behavior is obtained in the small signal conditions, followed by clipping (soft envelope limiting) at saturation. We focus on the SEL HPA model in this article. For the SEL model, the AM-AM and AM-PM conversions are given by [21]

$$
g_{A}[r(t)]=\left\{\begin{array}{cc}
\alpha_{A} r(t), & 0 \leq r(t) \leq A_{\text {is }} \\
A_{\text {is }}, & r(t)>A_{\text {is }}
\end{array} g_{p}[r(t)]=0\right.
$$

where $\alpha_{A}$ is the small-signal gain, and $A_{\text {is }}$ is the input saturation voltage of the HPA. Let $x(t)$ denote the input signal into the HPA, and we assume that $x(t)$ is Gaussian distributed. Therefore, according to the Bussgang's theorem, the output of the HPA when the input is a Gaussian process is given as [22,23]

$$
\hat{x}(t)=k x(t)+w(t)
$$

where $k$ (i.e., $0 \leq k \leq 1$ ) is an attenuation factor for the linear part which represents the in-band distortion, and $w(t)$ is a nonlinear additive noise which represents the out-of-band distortion. $w(t)$ is a zero-mean complex Gaussian random variable (r.v), with the in-phase and quadrature components mutually iid, and with variance $\sigma_{w}^{2}$. The in-band and out-of-band distortion terms can be calculated as [24]

$$
\begin{aligned}
& k=\frac{E\left\{x(t) \hat{x}^{*}(t)\right\}}{E\left\{x(t) x^{*}(t)\right\}}=\frac{1}{P_{\text {avg }}}\left(\int_{0}^{\infty} r \hat{x}^{*}(r) f(r) d r\right)^{*} \\
& \sigma_{w}^{2}=\frac{E\left\{\left|\hat{x}^{*}(r)\right|^{2}\right\}-|k|^{2}}{E\left\{|x|^{2}\right\}}=\frac{1}{P_{\text {avg }}}\left(\int_{0}^{\infty}\left|\hat{x}^{*}(r)\right|^{2} f(r) d r-|k|^{2}\right)
\end{aligned}
$$

where '*' denotes complex conjugation, $P_{\text {avg }}=E\left[|x|^{2}\right]$ is the average input energy per symbol, and $f(r)$ is the probability density function (PDF) of the envelope of the input signal into the HPA (i.e., $r=|x|)$. The PDF of ' $r$ ' is Rayleigh distribution (since $x(t)$ is assumed Gaussian). The closedform expressions for the in-band and out-of-band distortion parameters for the SEL HPA model are calculated by substituting $f(r)$ into Equations (6) and (7) to obtain

$$
\begin{aligned}
& k_{j}=1-e^{-\gamma_{j}^{2}}+\frac{1}{2} \sqrt{\pi} \gamma_{j} \operatorname{erfc}\left(\gamma_{j}\right) \\
& \sigma_{w_{j}}^{2}=P_{j}\left(1-e^{-\gamma_{j}^{2}}-k_{j}^{2}\right) \\
& \gamma_{j}=\sqrt{I B O_{j}}=\frac{A_{\mathrm{is}, j}}{\sqrt{P_{j}}}
\end{aligned}
$$

where $j=0,1, \ldots, R$ denotes the number of relay hops, $\gamma_{j}$ represents the clipping ratio (CR), $A_{\mathrm{is}, j}$ represents the input 
saturation voltage of each and every HPA employed at the $j$ th relay hop, and $P_{j}$ represents the average input power into the HPA's at the $j$ th hop. The frequency domain (FD) expression of the signal above is then obtained by taking its discrete Fourier Transform (DFT).

\section{Effect of HPA nonlinearity on the spectrum of MIMO-OFDM relaying system}

PSD of the nonlinearly amplified OFDM signal at the BS

OFDM signal is well approximated by zero-mean complex Gaussian signals for the large number of subcarriers [3]. Using the Bussgang theorem, the output of the HPA at the MIMO-OFDM BS transmitter is given by

$$
\hat{\mathbf{x}}(t)=\mathrm{K}_{0} \mathbf{x}(t)+\mathbf{w}_{0}(t)
$$

where the vector $\hat{x}(t)=\left[\hat{x}^{1}(t), \ldots, \hat{x}^{N}(t)\right]$ and $\hat{x}^{j}(t)$ are the output of the HPA at the $j$ th transmitting antenna of the BS when the input to the amplifier is $x^{j}(t) . K_{0}$ is an $N \times N$ diagonal matrix, where $k_{0}^{j}$ represents the scaling factor of the linear part (in-band distortion) at the $j$ th transmit antenna of the BS. $\mathbf{w}_{0}(t)=\left[w_{0}^{1}(t), \ldots, w_{0}^{N}(t)\right]$, and $w_{0}^{j}(t)$ is the nonlinear distortions noise (out-of-band distortion) due to HPA at the $j$ th transmitting antenna of the BS. The PSD of a signal is commonly estimated by computing the autocorrelation function of the signal followed by a Fourier transform, using the well-known Wiener-Khintchin theorem [25]. The PSD of the OFDM signal at the output of the nonlinear HPA at the $j$ th transmit antenna of a MIMO-OFDM transmitter is given by

$$
P_{\hat{x} \hat{x} \hat{x}^{j}}^{j}(f)=\int_{-\infty}^{+\infty} \Phi_{\hat{x} \hat{x} \hat{x}^{j}}^{j}(\tau) e^{-j 2 \pi f \tau} d \tau
$$

where $P_{\hat{x}^{\prime} \hat{x}^{j}}^{j}(f)$ and $\Phi_{\hat{x}^{\prime} \hat{x}^{j}}^{j}(\tau)$ represent the PSD and autocorrelation function of the OFDM signal at the output of the HPA at the jth transmitting antenna. The autocorrelation function for the output of the HPA at the jth transmitting antenna can be computed as

$$
\begin{aligned}
\Phi_{\hat{x}^{j}} \hat{x}^{j}(\tau) & =\lim _{T \rightarrow \infty} \frac{1}{2 T} \int_{-T}^{T} \hat{x}^{j}(t)\left[\hat{x}^{j}(t+\tau)\right]^{*} d t \\
& =E\left\{\hat{x}^{j}(t)\left[\hat{x}^{j}(t+\tau)\right]^{*}\right\} \\
& =E\left\{\left[k_{0}^{j} x^{j}(t)+w_{0}^{j}(t)\right]\left[k_{0}^{j} x^{j}(t+\tau)+w_{0}^{j}(t+\tau)\right]^{*}\right\} \\
& =\left|k_{0}^{j}\right|^{2} E\left\{x^{j}(t)\left[x^{j}(t+\tau)\right]^{*}\right\}+E\left\{w_{0}^{j}(t)\left[w_{0}^{j}(t+\tau)\right]^{*}\right\} \\
& =\left|k_{0}^{j}\right|^{2} \Phi_{\mathbf{x}^{\prime} x^{j}}(\tau)+\Phi_{\mathbf{w}_{0}^{j} \mathbf{w}_{0}^{j}}(\tau)
\end{aligned}
$$

where $k_{0}^{j}$ is the attenuation factor of the in-band part, $\Phi_{x^{j} x^{j}(\tau)}$ represents the autocorrelation function of the OFDM signal at the input of the HPA, i.e., $x^{j}(t)$, and
$\Phi_{\mathbf{w}_{0}^{j} \mathbf{w}_{0}^{j}}(\tau)$ represents the autocorrelation function of the out-of-band nonlinear noise (i.e., $w^{j}(t)$ ), contributed by the HPA at the $j$ th transmitting antenna of the source node (BS or MS). Using Equations (12) and (13), the PSD of the OFDM signal at the output of the HPA at the $j$ th transmitting antenna of the source node can be written as

$$
P_{\hat{x} \hat{x} \hat{x}^{j}}^{j}(f)=\left|k_{0}^{j}\right|^{2} P_{x j}^{j}(f)+P_{w_{0}^{j} w_{0}^{j}}^{j}(f)
$$

From Equation (14), it is easily observed that the PSD of the OFDM signal at the output of the HPA at the BS is attenuated by a factor of $\left|k_{0}^{j}\right|^{2}$, since $0 \leq k_{i}^{j} \leq 1$. It is also observed from this equation that the PSD of the OFDM signal at the output of the HPA is broadened, compared to the PSD at the HPA input, by a factor of $P_{w_{0}^{j} w_{0}^{j}}^{j}(f)$. This spectral broadening term contributed by the $j$ th transmit antenna, $P_{w_{0}^{j} w_{0}^{j}}^{j}(f)$, is related to the corresponding nonlinear noise variance $\sigma_{w_{0}^{j}}^{j}$ as

$$
{\sigma_{w_{0}^{j}}^{j}}^{2}=\int_{-\infty}^{+\infty} P_{w_{0}^{j} w_{0}^{j}}^{j}(f) d f
$$

Using the result from [26, Equation (16)], Equation (15) can be approximated as $\sigma_{w_{0}^{j}}^{j} \approx P_{w_{0}^{j} w_{0}^{j}}^{j}(f) B$, where $B$ denotes the bandwidth of the signal. This approximation is valid if the spectrum of $w_{0}^{j}$ is flat across the bandwidth, which is true for the subcarrier-based analysis considered here. As the variance of the nonlinear noise due to HPA increases, the spectral re-growth of the transmitted OFDM signal increases as can be observed from Equation (15). Since the analysis above gives estimate of the spectral re-growth due to HPA per transmitting antenna, then for MIMO-OFDM system with $N$ transmitting antennas, the overall spectral re-growth that occur due to HPA nonlinearity is given by $P_{w_{0} w_{0}}^{\mathrm{MIMO}}=\sum_{j=1}^{N} P_{w_{0}^{j} w_{0}^{j}}^{j}(f)$.

\section{PSD of the nonlinearly amplified OFDM signals at RS's}

Next we calculate the PSD of the nonlinearly amplified OFDM signals at RS's. For this analysis, we consider two cases. In the first case, we consider that each RS has ability to perform MIMO signal processing on the received signal before amplifying and forwarding it onto the next hop, and thus we could examine the spectrum of the OFDM symbols obtained at each receiving antennas of the RS. This case corresponds to the DemF relaying option. In the second case, we consider that the RS does not have ability to perform MIMO signal processing on the received signal before forwarding onto the next hop. The RS simply AF the received OFDM 
signals at the RF stage without demodulating the OFDM signal. This case corresponds to the AF relaying option. AF has the best latency performance, and is attractive for broadband transmissions over relaying channels. The case of DF relaying option where RS actually decode data transmitted on each OFDM subcarriers before reencoding/forwarding it is not analyzed here because the existing single-hop analyses in [27] and other references are valid for that case using hop-by-hop analysis. However, we later include the DF case in the simulation results as a reference.

\section{Case I: DemF relaying system}

In DemF relaying system, the received signal at the first RS for the $l$ th subcarrier is given by

$$
\begin{aligned}
Y_{R 1}[l] & =H_{0}[l] \hat{X}[l]+N_{0}[l] \\
& =K_{0} H_{0}[l] X[l]+H_{0}[l] W_{0}[l]+N_{0}[l]
\end{aligned}
$$

where $X[l]=\left[X^{1}[l] X^{2}[l] \cdots X^{N}[l]\right] T$ is an $N \times 1$ input OFDM signal in FD at the BS, and $X^{j}[l]$ is the input into the HPA at the $j$ th transmitting antenna on the $l$ th subcarrier. $\mathbf{K}_{i}=\operatorname{diag}\left\{k_{i}^{j}\right\}_{j=1}^{N}, i=0, \ldots, R$ is an $N \times N$ diagonal matrix, where $k_{i}^{j}$ represents the scaling factor of the linear part (in-band distortion) at the $j$ th transmit antenna in the $i$ th hop. $W[l]=\left[\begin{array}{llll}W_{i}^{1}[l] W_{i}^{2}[l] & \cdots & \left.W_{i}^{N}[l]\right]^{T} \text { is an } N \times 1\end{array}\right.$ nonlinear distortion noise vector (out-of-band distortion) due to HPA and it is obtained by taking the DFT of $\mathbf{w}(t)$. $W_{i}^{j}[l]$ represents the nonlinear distortion noise on the $j$ th transmit antenna in the $i$ th hop for the $l$ th

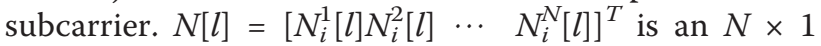
complex additive noise vector and $N_{i}^{j}[l]$ represents the iid zero-mean complex AWGN noise on the $j$ th transmit antenna in the $i$ th hop for the $l$ th subcarrier. $Y_{R_{1}}[l]=\left[Y_{R_{1}}^{1}[l] Y_{R_{1}}^{2}[l] \ldots Y_{R_{1}}^{N}[l]\right]^{T}$ is an $N \times 1$ received OFDM symbol vector in FD at the first RS and $Y_{R_{1}}^{j}[l]=$ $\sum_{m=1}^{N} H_{j m}^{0}[l] \hat{X}_{m}[l]+N_{0}^{j}[l]$ is the received OFDM symbol at the $j$ th received antenna of the first RS for the $l$ th subcarrier. We assume that the channel between the BS and the RS is known at the RS. Thus, we can employ MIMO Zero-Forcing or MIMO minimum mean square error technique for the MIMO signal processing at the RS for the DemF relaying option as

$$
Y_{R_{1}, \operatorname{DemF}}[l]=\mathbf{G}_{0}[l] Y_{R_{1}}[l]
$$

where

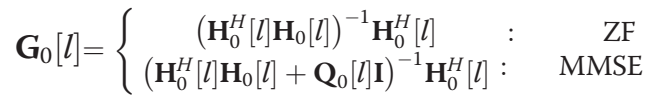

and $\mathbf{Q}_{0}[l]=E\left[\left(\mathbf{H}_{0}[l] W_{0}[l]+N_{0}[l]\right)\left(\mathbf{H}_{0}[l] W_{0}[l]+N_{0}[l]\right)^{H}\right]$. Here, we used the ZF technique for simplicity. The received symbol at the input of the first RS's HPA can then be expressed as

$$
\begin{aligned}
Y_{R_{1}, \operatorname{DemF}}[l] & =K_{0} X[l]+W_{0}[l]+G_{\mathrm{ZF}}[l] N_{0}[l] \\
& =K_{0} X[l]+W_{0}[l]+N_{0}^{\prime}[l]
\end{aligned}
$$

where $Y_{R_{1}, \text { DemF }}[l]=\left[Y_{R_{1} \text {,DemF }}^{1}[l] Y_{R_{1}, \text { DemF }}^{2}[l] \ldots Y_{R_{1}, \text { DemF }}^{N}[l]\right]^{T}$ is an $N \times 1$ received OFDM symbol vector in FD at the input of the RS's HPA, and $Y_{R_{1} \text {,DemF }}^{j}[l], l=1,2, \ldots, n$ is the input symbol at the $l$ th subcarrier and $j$ th received antenna of the RS. Also, we use $N_{0}^{\prime}[l]=\mathbf{G}_{Z F}[l] N_{0}[l]$ for notational convenience. The received OFDM signals are then amplified (normalized) separately on each subcarrier with an amplification factor $\alpha$, where $\alpha$ is an $n N \times n N$ block diagonal matrix whose diagonal entries for the $l$ th subcarrier and $i$ th hop transmission is given by $\operatorname{diag}\left\{\alpha_{i}^{1}[l], \alpha_{i}^{2}[l], \ldots\right.$, $\left.\alpha_{i}^{N}[l]\right\}, l=1, \ldots, n$. There are many choices for the selection of the amplification parameter $\alpha$ in relaying systems $[5,20]$. Sulyman et al. [20] amplify all the subcarriers in an OFDM symbol with the same parameter $\alpha$ without decoding the symbols. Riihonen et al. [5] demodulate the OFDM symbols at the RS to amplify each subcarrier separately with the amplification parameter $\alpha$. The amplification factor for the $k$ th subcarrier is selected to satisfy the transmit power constraint at the RS as

$$
\alpha_{l}^{j}[k]=\sqrt{\frac{P_{R}}{E\left[\left|y_{R_{l}, \text { DemF }}^{j}[k]\right|^{2}\right]}}
$$

where $P_{R}$ is the total transmit power available at the RS. Before transmitting onto the next hop, the OFDM symbols are passed through the HPA at the RS as shown in Figure 2. The output of the HPA at the $j$ th transmit antenna of the first RS $\left(R_{1}\right)$ in time domain (TD) is given by

$$
\begin{aligned}
\hat{\mathbf{y}}_{R_{1}, \text { DemF }}^{j}(t)= & \alpha_{1}^{j} k_{0}^{j} k_{1}^{j} x^{j}(t)+\alpha_{1}^{j} k_{1}^{j} w_{0}^{j}(t) \\
& +\alpha_{1}^{j} k_{1}^{j} n_{0}^{\prime j}(t)+w_{1}^{j}(t)
\end{aligned}
$$

where $n_{0}^{\prime j}(t)$ and $w_{0}^{j}(t)$ are, respectively, the thermal noise and the nonlinear noise of the HPA, propagated from the first hop transmission. $w_{1}^{j}(t)$ and $k_{1}^{j}$ are the HPA distortion terms introduced by the first RS. To calculate the PSD of the output of the HPA at the $j$ th transmit antenna of the first RS, we calculate the autocorrelation function of the output of the HPA at the $j$ th transmit antenna of the RS as 


$$
\begin{aligned}
& \Phi_{R_{1} R_{1}}^{j}(\tau)=E\left\{\hat{y}_{R_{1}, \text { DemF }}^{j}(t)\left[\hat{y}_{R_{1}, \operatorname{DemF}}^{j}(t+\tau)\right]^{H}\right\} \\
& =E\left\{\begin{array}{c}
{\left[\alpha_{1}^{j} k_{0}^{j} k_{1}^{j} x^{j}(t)+\alpha_{1}^{j} k_{1}^{j} w_{0}^{j}(t)+\alpha_{1}^{j} k_{1}^{j} n^{\prime}{ }_{0}(t)+w_{1}^{j}(t)\right]} \\
\times\left[\alpha_{1}^{j} k_{0}^{j} k_{1}^{j} x^{j}(t+\tau)+\alpha_{1}^{j} k_{1}^{j} w_{0}^{j}(t+\tau)+\alpha_{1}^{j} k_{1}^{j} n^{\prime}{ }_{0}(t+\tau)+w_{1}^{j}(t+\tau)\right]^{H}
\end{array}\right\} \\
& =\left(\alpha_{1}^{j}\right)^{2}\left|k_{0}^{j}\right|^{2}\left|k_{1}^{j}\right|^{2} \Phi_{x^{j} x^{j}}(\tau)+\left(\alpha_{1}^{j}\right)^{2}\left|k_{1}^{j}\right|^{2} \Phi_{w_{0}^{j} w_{0}^{j}}(\tau)+\frac{\left(\alpha_{1}^{j}\right)^{2}\left|k_{1}^{j}\right|^{2} \Phi_{n_{0}^{j} n_{0}^{j}}(\tau)}{N}+\Phi_{w_{1}^{j} w_{1}^{j}}(\tau)
\end{aligned}
$$

where

$$
\begin{aligned}
E\left[\mathbf{G}_{i} \mathbf{G}_{i}^{H}\right] & =E\left\{\left[\left(\mathbf{H}_{i}^{H} \mathbf{H}_{i}\right)^{-1} \mathbf{H}_{i}^{H}\right]\left[\left(\mathbf{H}_{i}^{H} \mathbf{H}_{i}\right)^{-1} \mathbf{H}_{i}^{H}\right]^{H}\right\} \\
& =E\left\{\left[\mathbf{H}_{i}^{H} \mathbf{H}_{i}\right]^{-1}\right\} \\
& =\frac{1}{N} I_{N}
\end{aligned}
$$

Thus, the PSD of the OFDM signal at the $j$ th antenna of the RS is computed from the autocorrelation function using Wiener-Khintchin theorem and is given as

$$
\begin{aligned}
P_{R_{1} R_{1}, \text { DemF }}^{j}(f)= & \left(\alpha_{1}^{j}\right)^{2}\left|k_{0}^{j}\right|^{2}\left|k_{1}^{j}\right|^{2} P_{x^{j} j^{j}}(f) \\
& +\left(\alpha_{1}^{j}\right)^{2}\left|k_{1}^{j}\right|^{2} P_{w_{0}^{j} w_{0}^{j}}(f) \\
& +\frac{\left(\alpha_{1}^{j}\right)^{2}\left|k_{1}^{j}\right|^{2} P_{n_{0}^{j} n_{0}^{j}}(f)}{N} \\
& +P_{w_{1}^{j} w_{1}^{j}}(f)
\end{aligned}
$$

In general, for $R$ relay hops, we can repeat the steps in Equations (17)-(23) to obtain the spectrum of the transmitted OFDM signal at the $j$ th transmit antenna of the $R$ th RS as

$$
\begin{aligned}
P_{R R, \text { DemF }}^{j}(f)= & \left\{\left(\alpha_{1}^{j}\right)^{2} \cdots\left(\alpha_{R}^{j}\right)^{2}\right\} \prod_{l=0}^{R}\left|k_{l}^{j}\right|^{2} P_{\mathbf{x}^{j} \mathbf{x}^{j}}(f) \\
& +\left\{\left(\alpha_{1}^{j}\right)^{2} \cdots\left(\alpha_{R}^{j}\right)^{2}\right\} \sum_{m=0}^{R-1 R-m} \prod_{n=1}^{R-m}\left|k_{R+1-n}^{j}\right|^{2} P_{\mathbf{w}_{m}^{j} \mathbf{w}_{m}^{j}}(f) \\
& +\left\{\left(\alpha_{1}^{j}\right)^{2} \cdots\left(\alpha_{R}^{j}\right)^{2}\right\} \frac{1}{N} \sum_{p=0}^{R-1} \prod_{q=1}^{R-p}\left|k_{R+1-q}^{j}\right|^{2} P_{\mathbf{n}_{p}^{j} \mathbf{n}_{p}}(f) \\
& +P_{\mathbf{w}_{R}^{j} \mathbf{w}_{R}^{j}}(f)
\end{aligned}
$$

From the above expression, it can be seen that the spectral re-growth at the RS depends both on the nonlinear distortion noise due to HPA and on the thermal AWGN noise. For the case when we assume the same HPA characteristic at the BS and $R$ RS, then $\mathbf{K}_{0}=$ $\mathbf{K}_{1}=\cdots=\mathbf{K}_{R}=\mathbf{K}$, and if we assume the same amplification factor at all $R$ RS so that $\boldsymbol{\alpha}_{1}=\boldsymbol{\alpha}_{2}=\cdots=$ $\boldsymbol{\alpha}_{R}=\boldsymbol{\alpha}$, then Equation (24) can be expressed as

$$
\begin{aligned}
P_{R R, \text { DemF }}^{j}(f)= & \left(\alpha^{j}\right)^{2 R}\left(\left|k^{j}\right|\right)^{2(R+1)} P_{\mathbf{x}^{j} \mathbf{x}^{j}}(f) \\
& +\left(\alpha^{j}\right)^{2 R} \sum_{m=0}^{R-1}(|k|)^{2(R-m)} P_{\mathbf{w}_{m}^{j} \mathbf{w}_{m}^{j}}(f) \\
& +\frac{1}{N}\left(\alpha^{j}\right)^{2 R} \sum_{p=0}^{R-1}(|k|)^{2(R-p)} P_{\mathbf{n}_{p}^{j} \mathbf{n}_{p}^{j}}(f) \\
& +P_{\mathbf{w}_{R}^{j} \mathbf{w}_{R}^{j}}(f)
\end{aligned}
$$

\section{Case II: AF relaying system}

In AF relaying system, the received symbol at the input of the HPA of the first RS $\left(R_{1}\right)$ in FD is given by

$$
\begin{aligned}
Y_{R_{1}}[l] & =\mathbf{H}_{0}[l] \hat{X}[l]+N_{0}[l] \\
& =\mathbf{K}_{\mathbf{0}} \mathbf{H}_{0}[l] X[l]+\mathbf{H}_{0}[l] W_{0}[l]+N_{0}[l]
\end{aligned}
$$

where $Y_{R_{1}}[l]=\left[Y_{R_{1}}^{1}[l] Y_{R_{1}}^{2}[l] \ldots Y_{R_{1}}^{N}[l]\right]^{T} \quad$ is an $N \times 1$ received OFDM symbol vector, and $Y_{R_{1}}^{j}[l]$ is the received OFDM symbol at the $j$ th received antenna of $R_{1}$ for the $j$ th subcarrier and is given by

$$
\begin{aligned}
Y_{R_{1}}^{j}[l]= & \sum_{m=1}^{N} H_{j, m}^{0}[l] \hat{X}^{m}[m]+N_{0}^{j}[l] \\
= & \sum_{m=1}^{N} k_{0}^{m} H_{j, m}^{0}[l] X[m] \\
& +\sum_{m=1}^{N} H_{j, m}^{0}[l] W^{m}[l]+N_{0}^{j}[l]
\end{aligned}
$$

The received OFDM signal at the $j$ th receive antenna of $R_{1}$ is then amplified with an amplification factor $\alpha_{1}^{j}$ as shown in Figure 2b. The amplification 
parameter $\alpha_{1}^{j}[l]=\sqrt{\frac{P_{R_{1}}}{E\left[\left.Y_{R_{1}}[l]\right|^{2}\right]}}$ for the $j$ th antenna on the lth subcarrier is again selected such that the total transmitted power at $R_{1}$ is $P_{R_{1}}$. Before transmitting the OFDM signal onto the next hop, it is passed through the HPA at the RS. In general, the transmitted OFDM signal at the $m$ th RS $\left(R_{m}\right)$ in TD is given by

$$
\begin{aligned}
\hat{y}_{R_{m}, \mathrm{AF}}(t)= & \boldsymbol{\alpha}_{m} K_{m} \mathbf{H}_{m-1} \hat{y}_{R_{m-1}, \mathrm{AF}}(t) \\
& +\boldsymbol{\alpha}_{m} K_{m} \mathbf{n}_{m-1}(t)+\mathbf{w}_{m}(t)
\end{aligned}
$$

where $\hat{y}_{R_{m-1}, \mathrm{AF}}(t), m=1, \ldots, R$ is the transmitted OFDM symbol at the $(m-1)$ th RS, and the transmitted OFDM symbol at the output of the HPA at the $j$ th transmit antenna of the $m$ th RS is given by

$$
\begin{aligned}
\hat{y}_{R_{m}, A F}^{j}(t)= & \alpha_{m}^{j} k_{m}^{j} \sum_{l=1}^{N} h_{j l}^{m-1} \hat{y}_{R_{m-1}, \mathrm{AF}}^{j}(t) \\
& +\alpha_{m}^{j} k_{m}^{j} n_{m-1}^{j}(t)+w_{m}^{j}(t)
\end{aligned}
$$

For the case of $m=1$, i.e., one RS, Equation (29) can be written as

$$
\begin{aligned}
\hat{y}_{R_{m}, \mathrm{AF}}^{j}(t)= & \alpha_{1}^{j} k_{1}^{j} \sum_{l=1}^{N} k_{0}^{l} h_{j l}^{0} x^{l}(t)+\alpha_{1}^{j} k_{1}^{j} \sum_{l=1}^{N} h_{j l}^{l} w_{0}^{l}(t) \\
& +\alpha_{1}^{j} k_{1}^{j} n_{0}^{j}(t)+w_{1}^{j}(t)
\end{aligned}
$$

Then the PSD of the transmitted OFDM symbol at the output of the HPA at the $j$ th transmit antenna for the case $m=1$ is given as

$$
\begin{aligned}
P_{R_{1} R_{1}, \mathrm{AF}}^{j}(f)= & \left(\alpha_{1}^{j}\right)^{2}\left|k_{1}^{j}\right|^{2} \sum_{l=1}^{N}\left|k_{0}^{l}\right|^{2} P_{x^{l} x^{l}}(f) \\
& +\left(\alpha_{1}^{j}\right)^{2}\left|k_{1}^{j}\right|^{2} \sum_{l=1}^{N} P_{w_{0}^{l} w_{0}^{l}}(f) \\
& +\left(\alpha_{1}^{j}\right)^{2}\left|k_{1}^{j}\right|^{2} P_{n_{0}^{j} n_{0}^{j}}(f)+P_{w_{1}^{l} w_{1}^{l}}(f)
\end{aligned}
$$

Similarly, the general expression for the PSD of the transmitted OFDM symbol at the output of the HPA at the $j$ th transmitting antenna of the Pth RS is derived for $\mathrm{AF}$ relaying system as

$$
\begin{aligned}
P_{R_{P} R_{P}, \mathrm{AF}}^{j}(f)= & \left(\alpha_{P}^{j}\right)^{2}\left|k_{P}^{j}\right|^{2} \sum_{l=1}^{N}\left|k_{P-1}^{l}\right|^{2} P_{R_{P-1}^{l} R_{P-1}^{l}}(f) \\
& +\left(\alpha_{P}^{j}\right)^{2}\left|k_{P}^{j}\right|^{2} \sum_{l=1}^{N} P_{w_{P-1}^{l} w_{P-1}^{l}}(f) \\
& +\left(\alpha_{P}^{j}\right)^{2}\left|k_{P}^{j}\right|^{2} P_{n_{P-1}^{j} n_{P-1}^{j}}(f)+P_{w_{P}^{l} w_{P}^{l}}(f)
\end{aligned}
$$

where $P_{R_{P-1}^{l} R_{P-1}^{l}}(f)$ is the PSD of the transmitted OFDM symbol at the output of the HPA at the $l$ th transmitting antenna of the $(P-1)$ th RS.

\section{Simulation results and discussions}

In our simulation setup, we consider multihop MIMOOFDM system for $n=1024$ subcarriers and different number of transmit and receive antennas. We adopt 1024-FFT downlink sub-carrier allocation scheme defined in the WiMAX standard [16], as shown in Table 1. The HPA model employed in the simulation studies is the SEL model.

Figure 4 presents the time samples of the OFDM symbol we passed through the HPA in our simulation studies. It is evident from this figure that the OFDM signal has high amplitude variations (or high PAPR), thus some part of the signal will fall into the nonlinear regime of the HPA characteristics except if conservatively large back-off is used, which results in severe power penalty. A popular approach is to clip the high peaks in the OFDM signal to enable linear amplifications [27]. However, clipping operation itself is a nonlinear process and it results in in-band and out-of-band distortions. The SEL model employed in our simulation captures this HPA model. Figure 5 depicts the output of the HPA when the OFDM signal in Figure 4 is passed through HPA for different values of the CR. It is easy to observe from this figure that as the $\mathrm{CR}$ increases, the severity of the nonlinearity decreases and the time waveform of the output signal from the HPA approaches the input signal into the HPA. However, large clipping ratio incurs severe power penalties; therefore, there is a trade-off between HPA power efficiency and nonlinearities introduced.

Figure 6 shows the effect of HPA nonlinearity on the spectrum of OFDM signal for different CRs. For this simulation, we used the Welch's modified periodogram method for the estimation of the PSDs with the following parameters: $50 \%$ overlap between the segments and Hamming window. Welch function take into account the abrupt changes of the phase between the two successive symbols. It is observed from this figure that there is an in-band amplitude attenuation and an outof-band spectral re-growth observed in the spectrum of

Table 1 1024-FFT/IFFT parameters in $20 \mathrm{MHz}$ bandwidth

\begin{tabular}{ll}
\hline Parameter & Value \\
\hline Channel bandwidth & $20 \mathrm{MHz}$ \\
Modulation & $4-\mathrm{QAM}$ \\
Number of DC subcarriers & 1 \\
Number of guard subcarriers, left & 80 \\
Number of guard subcarriers, right & 79 \\
Number of data subcarriers & 864 \\
Subcarrier frequency spacing, $\Delta f$ & $19.53125 \mathrm{KHz}(=20 \mathrm{MHz} / 1024)$ \\
IFFT/FFT period, $T_{\mathrm{FFT}}$ & $51.2 \mu \mathrm{s}$ \\
Cyclic prefix duration, $T_{\mathrm{CP}}$ & $6.4 \mu \mathrm{s}\left(T_{\mathrm{FFT}} / 8\right)$ \\
Total OFDM symbol duration, $T_{\mathrm{S}}$ & $57.6 \mu \mathrm{s}$ \\
\hline
\end{tabular}




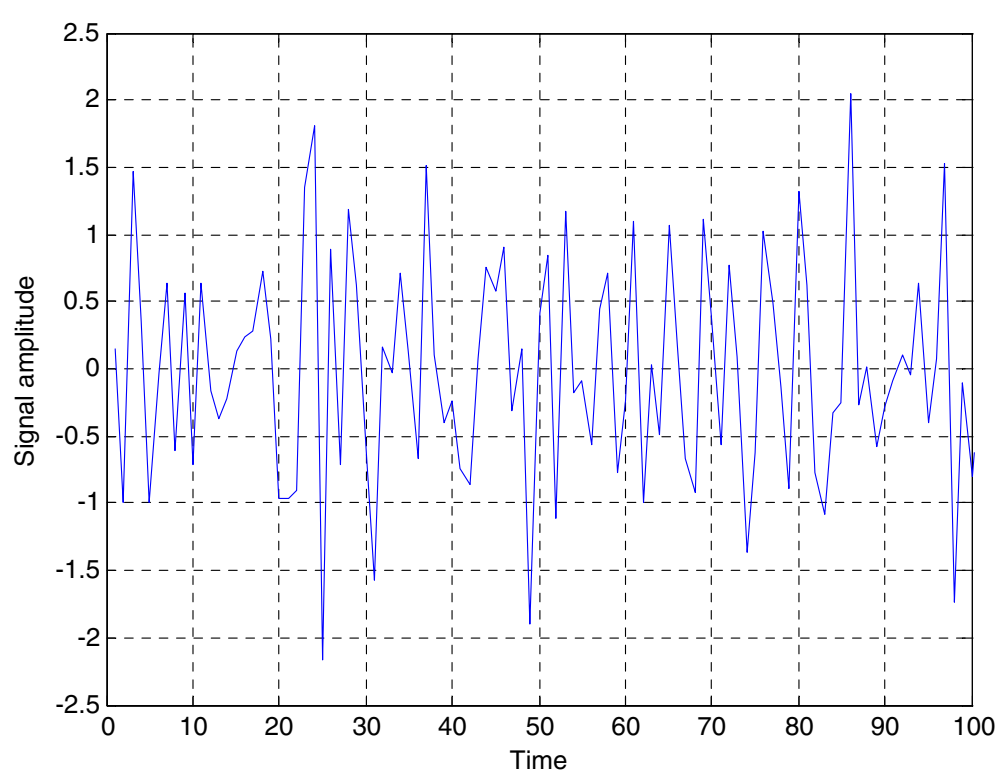

Figure 4 4-QAM, 1024 OFDM signal at the input of the HPA before clipping.

the output signal from the HPA, compared to its input. This confirms our earlier observations from the analysis. It is also observed from the figure that as we back-off the amplifier from the saturation region by increasing the CR, the out-of-band distortion decreases; however, the in-band amplitude attenuation increases resulting in more in-band signal power attenuation. This results in significant power penalty, making the use of large $\mathrm{CR}$ as a means of avoiding HPA nonlinearity unaffordable in practice in the cellular systems due to the need to transmit certain power levels in order to meet signal quality requirements at cell edges.
In [27], it is shown that at $C R=1.4 \mathrm{~dB}$, the distortion introduced by the HPA when transmitting OFDM signals through it compares favorably with the distortions encountered in a single-carrier QPSK signal using raisedcosine pulse shaping and a roll-off factor of 0.5 . This level of distortion is typically tolerable in many cellular systems, and hence $\mathrm{CR}=1.4$ is practically reasonable. We have thus used $\mathrm{CR}=1.4$ as a major reference in our simulation results presented next.

Figure 7 presents the spectrum of the OFDM signal obtained by simulation versus the spectrum obtained

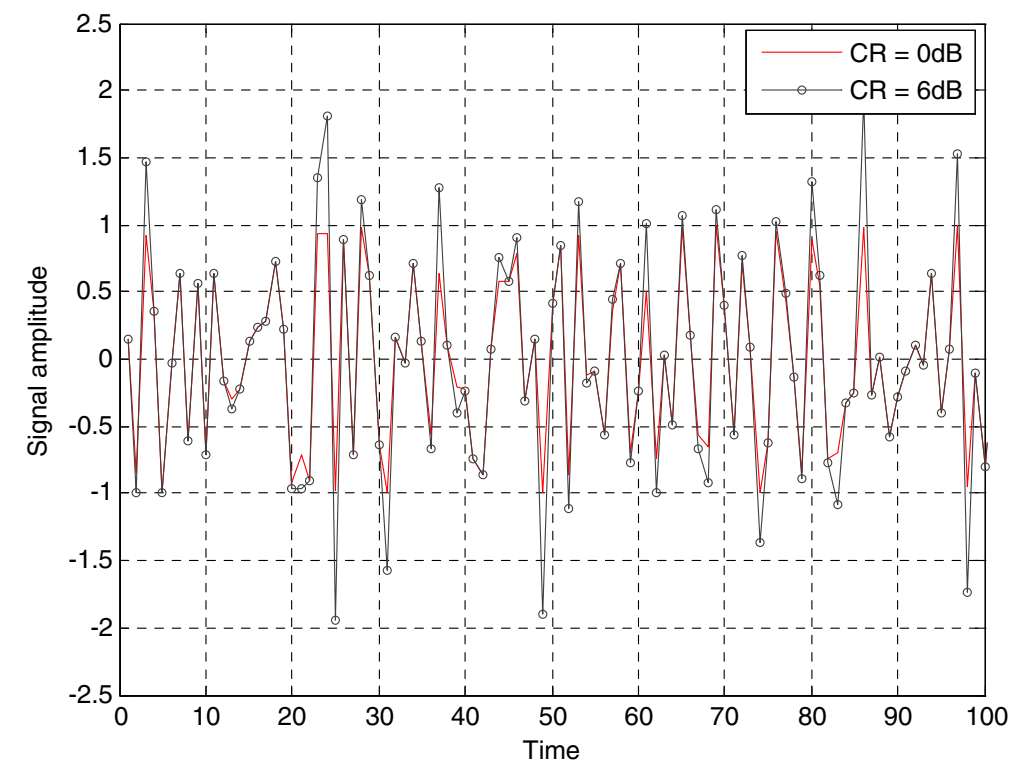

Figure 5 4-QAM, 1024 OFDM signal at the output of the HPA after clipping with CR of 0 and $6 \mathrm{~dB}$. 


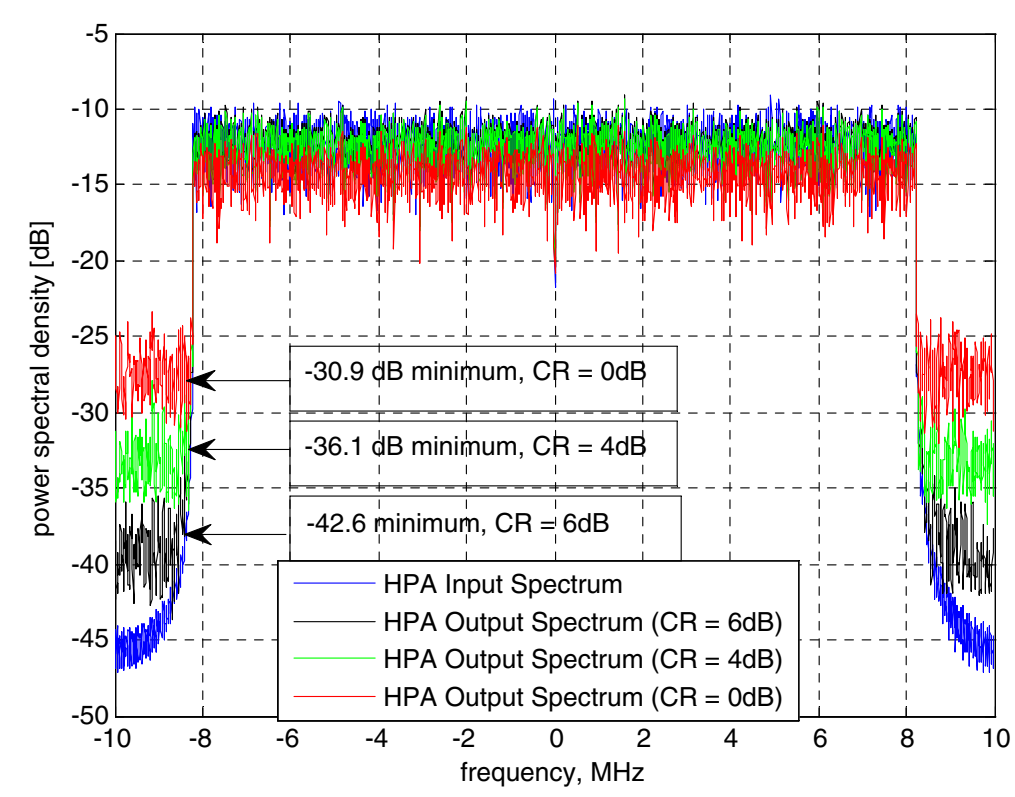

Figure 6 Effect of HPA nonlinearity on the spectrum of OFDM signals for different CRs at the BS.

from Equations (14) and (25). From Figure 7, it can be observed that both the analytical and simulation results agree very well as expected, and that both results indicate that significant spectral re-growth occur when the OFDM symbol is nonlinearly amplified at the BS and the RS. It is also observed that for $\mathrm{CR}=1.4 \mathrm{~dB}$, the gap between the main transmitted signal power and the outof-band spectral re-growth at $1.4 \mathrm{MHz}$ offset is roughly
$(25-10)=15 \mathrm{~dB}$ at the RS and $(33-10)=23 \mathrm{~dB}$ at the BS. This implies that while the WiMAX spectral mask is fairly satisfied at the output of HPA with $\mathrm{CR}=1.4 \mathrm{~dB}$ at the BS, it is significantly violated at the RS, even for the 2-hop transmission shown in this figure.

Figure 8 presents the spectrum of the nonlinearly amplified OFDM signal at RSs, for different number of relay hops, using DemF relaying option and $C R=1.4$

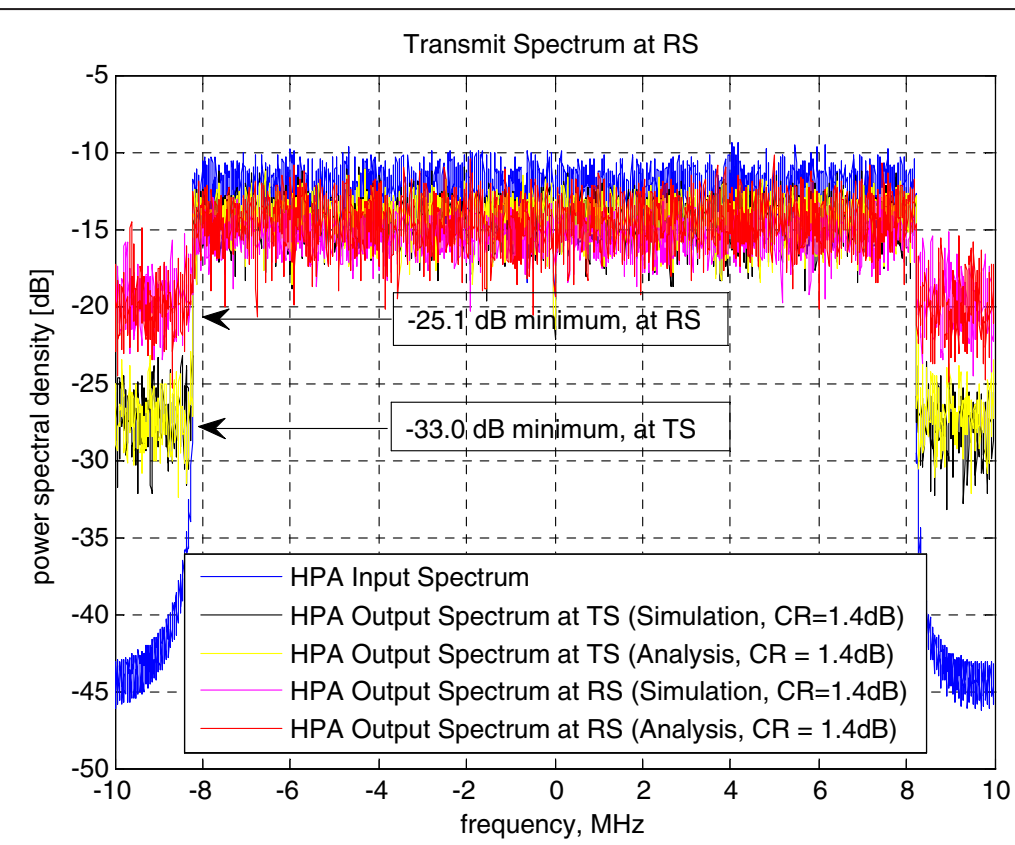

Figure 7 Comparison between analytical and simulation results for the spectrum of OFDM signals at the output of the HPA at the BS and at the RS, SNR $=10 \mathrm{~dB}$ at RS. 


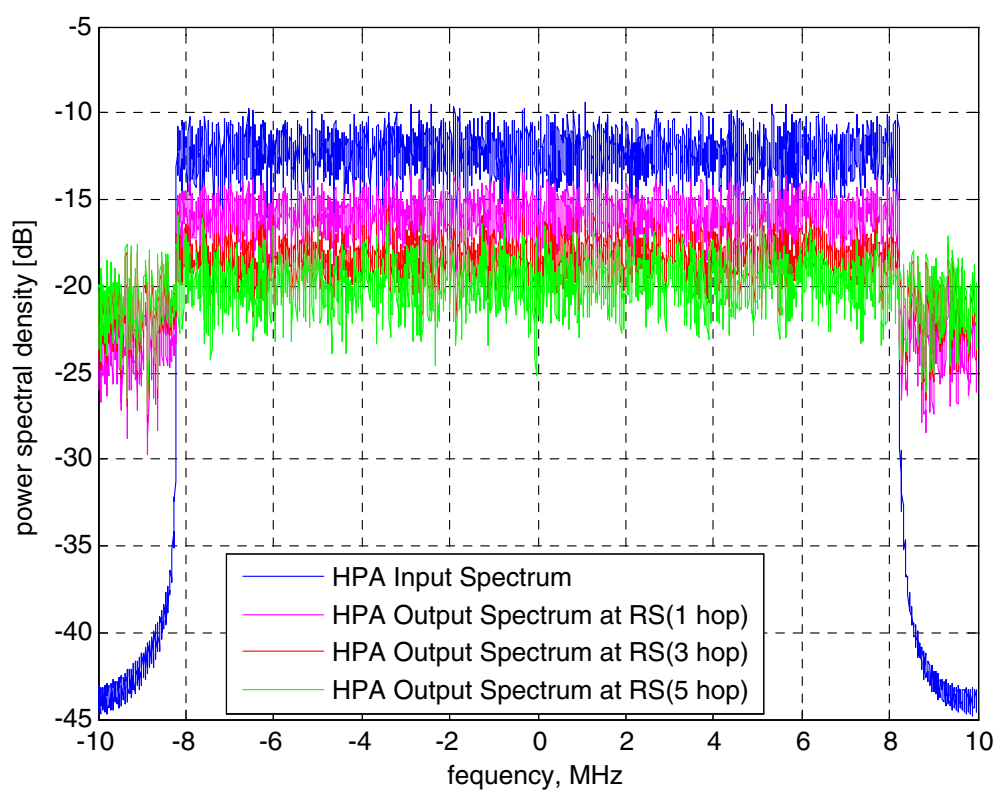

Figure 8 Effect of HPA nonlinearity on the spectrum of OFDM signals for different number of relay hops (DemF, CR $=1.4 \mathrm{~dB}, \mathrm{SNR}=15 \mathrm{~dB}$ ).

dB. It is observed from these results that the out-of-band distortion (i.e., spectrum re-growth in adjacent band) increases as the OFDM signal is relayed across multiple RSs, as observed earlier from the analytical results in Equation (25). Thus, the WiMAX spectral mask displayed in Figure 1 would easily be violated when large numbers of relay hops are involved. Figure 9 compares the effect of HPA nonlinearity on the spectrum of the relayed OFDM signals using AF and DemF relaying configurations. The spectrum of the relayed OFDM signals using DF relaying option is also included in the figure for reference. As expected, it can be observed from this figure that there is a higher spectral re-growth in AF relay option than in the DemF relay option. Also comparison between the spectral re-growth in DF relay option with DemF and AF relay options, for different

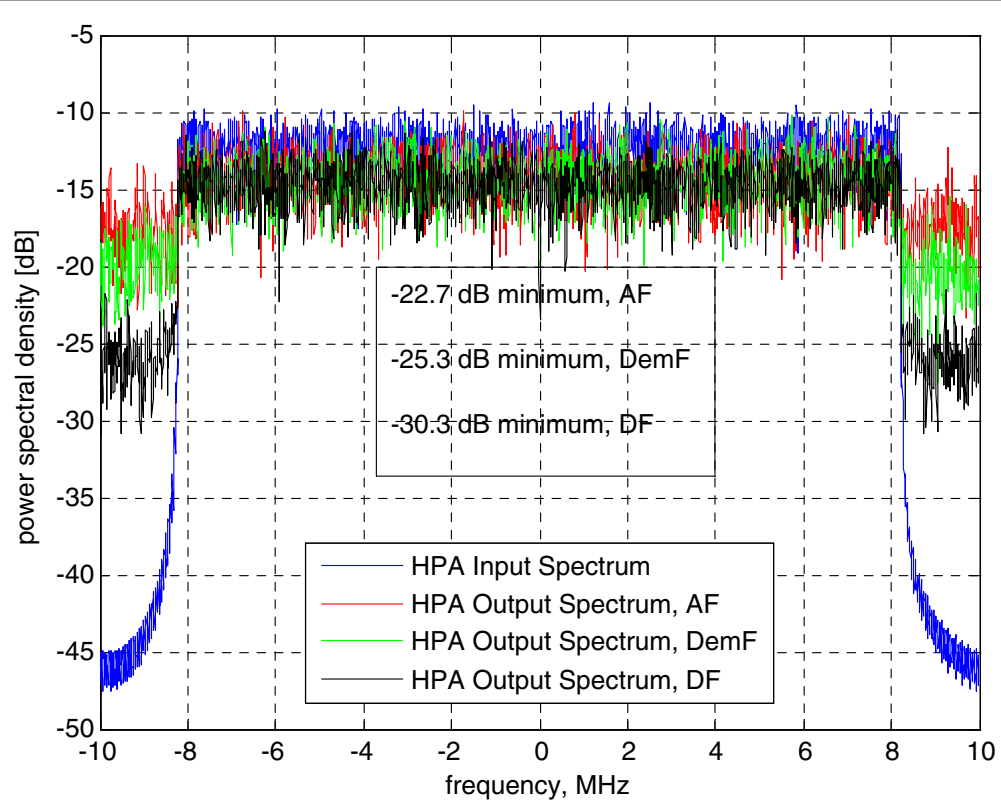

Figure 9 Effect of HPA nonlinearity on the spectrum of OFDM signals for AF, DemF, and DF configurations at RSs $(C R=1.4 \mathrm{~dB}, \mathrm{SNR}=10 \mathrm{~dB})$. 
values of SNR at the RS, shows that less severe spectral broadening is observed for the DF relaying option than the other two. It should be noted that these results were evaluated from the analytical expressions derived above, which hold for any CR. Therefore, the relative performance of DF, DemF, and AF relay options depicted in this figure hold also for other values of CR.

Next we present results for the case when we window the OFDM signal using linear-phase finite-impulse-response (FIR) digital filter before passing it through the nonlinear HPA, similar to the set up used for the works in $[15,28-30]$. Since the analysis did not model the effect of filtering, this part of the study is conducted solely by simulations. We use band-pass equi-ripple FIR filter with 110 coefficients and pass-band $0.2 \leq \omega \leq 0.6$, where $\omega=1$ corresponds to the Nyquist frequency, for our simulation. Figure 10 shows the effect of HPA nonlinearity on the spectrum of filtered OFDM signal for different values of CR. It is observed that because of the attenuation of the filter in this case, the spectral regrowth decreases in filtered MIMO-OFDM system compared to the unfiltered case in Figure 6. However, the cumulative remnant re-growth is still significant considering the fact that the results in Figure 10 show what happens per MIMO antenna of the transmitting station, and there are total of $N$ antennas per node. The spectrum of the nonlinear OFDM signal for different number of relaying hops for DemF relaying is presented in Figure 11. It is observed from these results that the out-of-band distortion (i.e., spectrum re-growth in adjacent band) increases as the OFDM signal is relayed across multiple RSs. For example, for $N \times N$ MIMO-
OFDM system with 2-hop relaying, the observed spectral re-growth from Figure 11 will be

$$
P_{w_{2} w_{2}}^{\mathrm{MIMO}}=\left[10 \log _{10}(N)+(32-10) \mathrm{dB}\right]
$$

which can result in significant spectral mask violation as the number of relay hops increases. The result in this figure suggests that the WiMAX spectral mask will be violated once the transmitted signal is relayed beyond two hops since according to Equation (33), $P_{w_{2} w_{2}}^{\mathrm{MIMO}}=$ $\left[10 \log _{10}(2)+(32-10) \mathrm{dB}\right]>25 \mathrm{~dB}$, which means that a 25- $\mathrm{dB}$ gap between the main carrier power and the unwanted spectral re-growth cannot be achieved once the number of relay hops involved are more than two. Hence, we can draw a conclusion that even though the general mobile multihop relaying (MMR) system where data could traverse any number of relay hops from source to destination has widely been studied theoretically [20,31,32], only the 2-hop version such as those proposed by the IEEE 802.16j group [18] may be advised for any practical deployments in MIMO-OFDM transmissions because of the potentials for spectral mask violations when going beyond 2-hop transmissions as shown in these results. In Figure 12, the effect of HPA nonlinearity on DF-relayed OFDM signal is presented. It can be observed from this figure that less severe spectral broadening is observed for the DF relaying option. However, due to the latency problems associated with the DF relaying option which degrades quality of broadband signals, AF or DemF are the preferred candidates for broadband transmissions over relaying channels and therefore the spectral broadening issues observed here

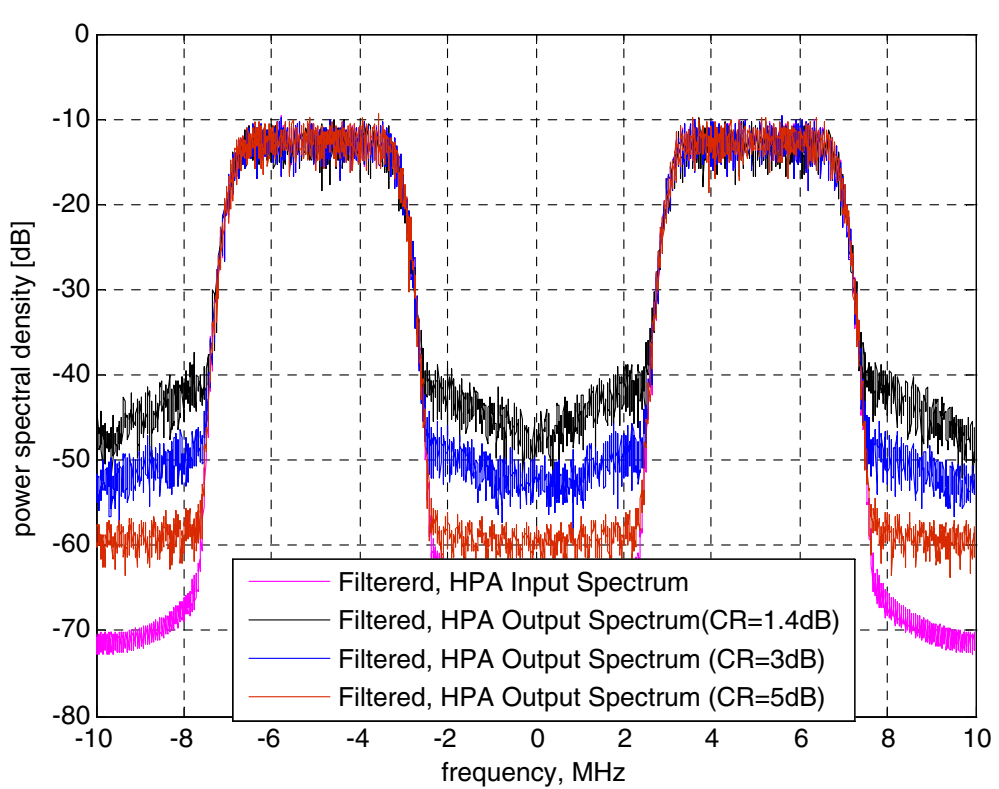

Figure 10 Effect of HPA nonlinearity on the spectrum of filtered OFDM signals at the BS for different CRs. 


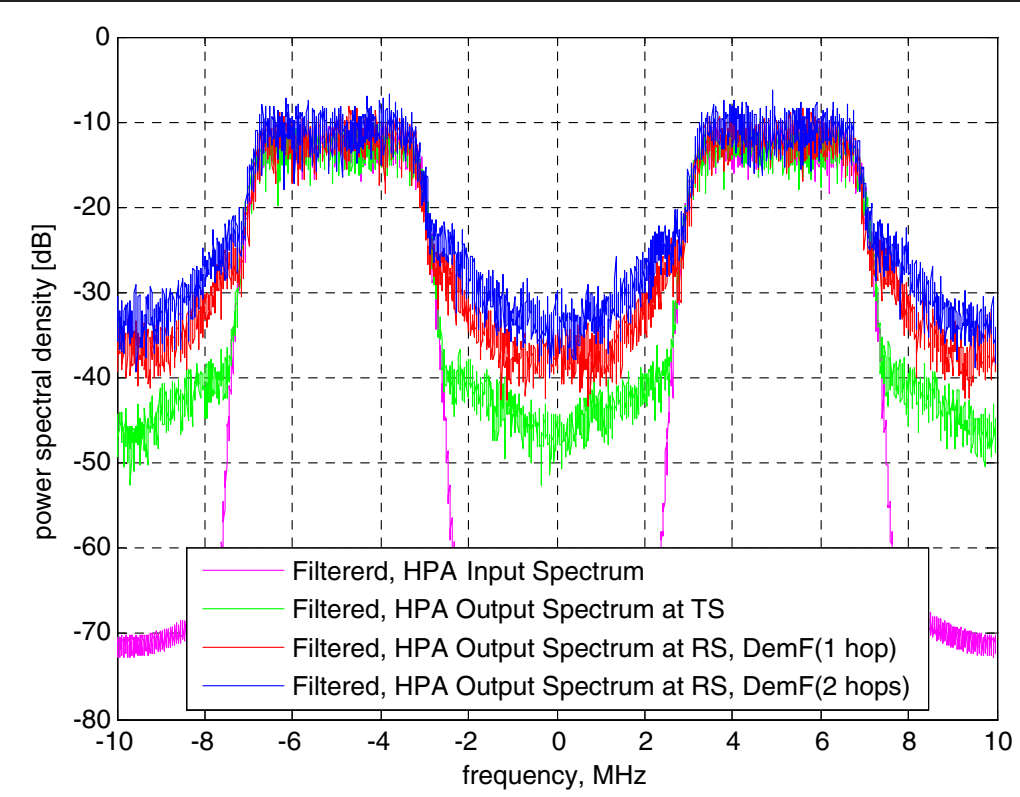

Figure 11 Effects of HPA nonlinearity on the spectrum of filtered OFDM signals in multihop relay channel $(C R=1.4 \mathrm{~dB}, \mathrm{SNR}=10 \mathrm{~dB}$ at each RS), DemF relaying at RSs.

must be given considerable attentions in the design of broadband multihop-relaying systems.

\section{Conclusions}

This article presents new insights on the out-of-band spectral re-growth due to HPA nonlinearities when MIMO-OFDM signals are transmitted over multiple relay channels. Expressions for the PSD of a MIMO-
OFDM signal are presented when it is transmitted from a BS to the MS via multiple RSs, all equipped with nonlinear HPAs. It is shown that significant spectral regrowth occurs for the AF and DemF relaying options as the OFDM signal traverse one or more relay hops. For MIMO-OFDM systems with large number of relay hops and MIMO antennas, the cumulative effects of this regrowth can result in significant spectral broadening

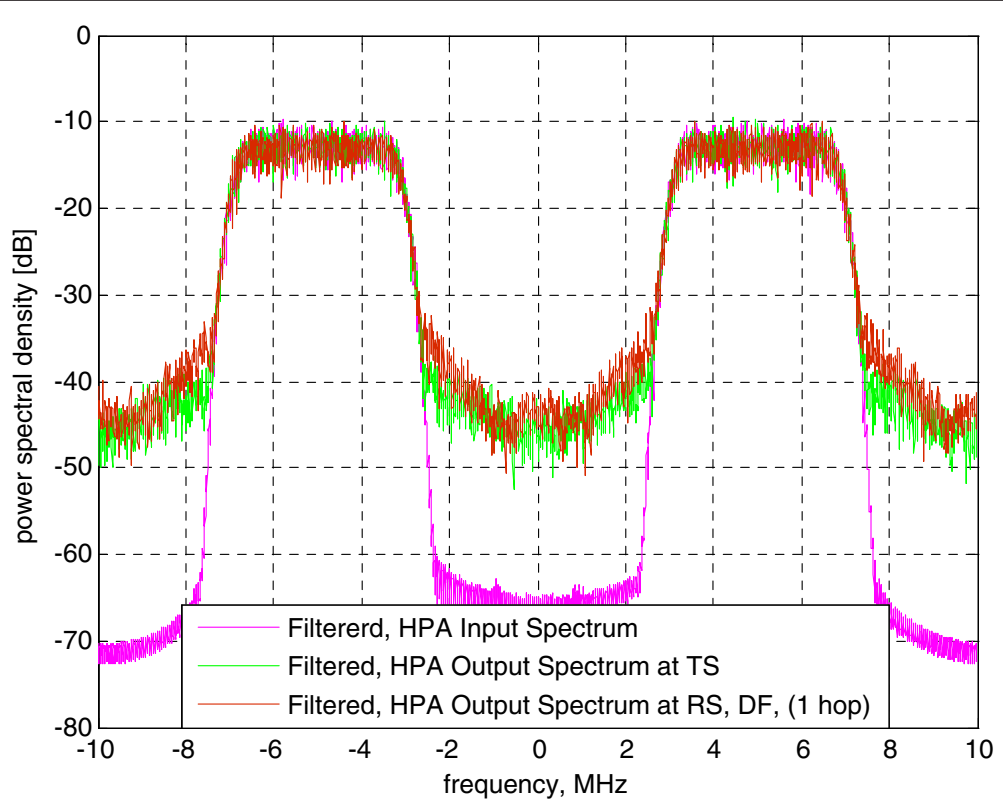

Figure 12 Effects of HPA nonlinearity on the spectrum of filtered OFDM signals in multihop relay channel $(C R=1.4 \mathrm{~dB}, \mathrm{SNR}=10 \mathrm{~dB}$ at each $\mathrm{RS})$, DF relaying at RSs. 
exceeding specified limits on the spectral masks. Hence, it is concluded from our results that even though the general MMR system where data could traverse any number of relay hops from source to destination have widely been studied theoretically, only the 2-hop version proposed by the IEEE $802.16 j$ group [18] may be advised for any practical deployments in MIMO-OFDM transmissions because of the potentials for spectral mask violations when going beyond 2-hop transmissions.

\section{Competing interests}

The authors declare that they have no competing interests.

\section{Acknowledgments}

This study was sponsored by a grant (No. 09-ELE928-02) from The National Plan for Science and Technology (NPST), King Saud University, Saudi Arabia.

\section{Author details}

${ }^{1}$ Department of Electrical Engineering, King Saud University, Riyadh, Saudi Arabia. ${ }^{2}$ KACST Technology Innovation Center RFTONICS, King Saud University, Riyadh, Saudi Arabia.

Received: 14 June 2012 Accepted: 10 November 2012 Published: 15 February 2013

\section{References}

1. S-H Wang, C-P Li, A low-complexity PAPR reduction scheme for SFBC MIMO-OFDM systems. IEEE Signal Process. Lett. 16(11), 941-944 (2009)

2. Y Wang, W Chen, C Tellembura, A PARP reduction method based on artificial bee colony algorithm for OFDM signals. IEEE Trans. Wirel. Commun. 9(10) (2010)

3. E Costa, S Pupolin, M-QAM-OFDM system performance in the presence of a nonlinear amplifier and phase noise. IEEE Trans. Commun. 50(3), 462-472 (2002)

4. Al Sulyman, M Ibnkahla, Performance analysis of nonlinearly amplified MQAM signals in MIMO channels. Eur. Trans. Telecommun. 19(1), 15-22 (2008)

5. T Riihonen, S Werner, F Gregorio, R Wichman, J Hamalainen, BEP analysis of OFDM relay links with nonlinear power amplifiers, in Proceedings of the 2010 IEEE Wireless Communications and Networking Conference (WCNC), Sydney, Australia, 2010, p. 6

6. J Qi, S Aissa, On the power amplifier nonlinearity in MIMO transmit beamforming systems. IEEE Trans. Commun. 60(3), 876-887 (2012)

7. PM Castro, JP Gonzalez-Coma, JA Garcia-Naya, L Castedo, Performance of MIMO systems in measured indoor channels with transmitter noise. EURASIP $J$. Wirel. Commun. Netw. no. 1, 19 (2012). doi:10.1186/1687-1499-2012-109

8. J Qi, S Aissa, Analysis and compensation of power amplifier nonlinearity in MIMO transmit diversity systems. IEEE Trans. Veh. Technol. 59(6), 2921-2931 (2010)

9. R Heung-Gyoon, System design and analysis of MIMO SFBC CI-OFDM system against the nonlinear distortion and narrowband interference. IEEE Trans. Consum. Electron. 54(2), 368-375 (2008)

10. I Ahmad, Al Sulyman, A Alsanie, A Alasmari, On the effect of amplifier nonlinearity on the capacity of MIMO systems, in Proceedings of the IEEE GCC Conference (GCC), Dubai, UAE, 2011, pp. 108-111

11. P Banelli, S Cacopardi, Theoretical analysis and performance of OFDM signals in nonlinear AWGN channels. IEEE Trans. Commun. 48(3), 430-441 (2000)

12. KG Grad, LE Larson, MB Steer, The impact of RF front-end characteristics on the spectral regrowth of communications signals. IEEE Trans. Microw. Theory 53(6), 2179-2186 (2005)

13. X Li, H Xiao, C Liu, F Li, Nonlinearity analysis of RF power amplified TDSCDMA signals, in Proceedings of the 10th IEEE International Conference on Signal Processing (ICSP), Beijing, China, 2010, pp. 1636-1641

14. E Cottais, Y Wang, S Toutain, Spectral regrowth analysis at the output of a memoryless power amplifier with multicarrier signals. IEEE Trans. Commun. 56(7), 1111-1118 (2008)

15. TK Helaly, RM Dansereau, M El-Tanany, An efficient measure for nonlinear distortion severity due to HPA in downlink DS-CDMA signals. EURASIP J. Wirel. Commun. Netw. no. 1, 9 (2010). doi:10.1155/2010/945427. article ID 945427

16. IEEE Standard for Local and Metropolitan Area Networks- Parts 16: Air Interface for Fixed and Mobile Broadband Wireless Access System, 2009
17. F Gregorio, J Cousseau, S Werner, T Riihonen, R Wichman, Power amplifier linearization technique with IQ imbalance and crosstalk compensation for broadband MIMO-OFDM transmitters. EURASIP J. Adv. Signal Process. no. 1, 15 (2011). doi:10.1186/1687-6180-2011-19

18. SW Peters, RW Heath, The future of WiMAX: multihop relaying with IEEE 802.16j. IEEE Commun. Mag. 47, 104-111 (2009)

19. A Ghosh, R Ratasuk, B Mondal, N Mangalvedhe, T Thomas, LTE-advanced: next-generation wireless broadband technology [Invited paper]. IEEE Trans. Wirel. Commun. 17(3), 10-22 (2010)

20. Al Sulyman, G Takahara, H Hassanein, M Kousa, Multi-hop capacity of MIMOmultiplexing relaying systems. IEEE Trans. Wirel. Commun. 8, 3095-3103 (2009)

21. FH Gregorio, Analysis and compensation of nonlinear power amplifier effects in multi-antenna OFDM systems (Ph.D. dissertation, Helsinki University of Technology, Espoo, Finland, 2007)

22. JJ Bussgang, Crosscorrelation functions of amplitude-distorted Gaussian signals, in Research Lab. Electron, M.I.T, Technical Report, Cambridge, MA, USA, 1952, p. 216

23. NY Ermolova, SG Haggman, An extension of Bussgang's theory to complexvalued signals, in Proceedings of the 6th Nordic Signal Processing Symposium (NORSIG'04), Espoo, Finland, 2004

24. F Gregorio, S Werner, TI Laakso, J Cousseau, Receiver cancellation technique for nonlinear power amplifier distortion in SDMA-OFDM systems. IEEE Trans. Veh. Technol. 56(5), 2499-2516 (2007)

25. JG Proakis, Digital Communications, 4th edn. (McGraw-Hill, New York, 2001)

26. DW Chi, P Das, Effects of nonlinear amplifiers and narrowband interference in MIMO-OFDM with application to $802.11 \mathrm{n}$ WLAN, in Proceedings of the 2nd International Conference on Signal Processing and Communication Systems (ICSPCS), Gold Coast, Australia, 2008, pp. 1-7

27. $X \mathrm{Li}, \mathrm{L} \mathrm{J}$ Cimini Jr, Effects of clipping and filtering on the performance of OFDM. IEEE Commun. Lett. 2(5), 131-133 (1998)

28. GR Tsouri, D Wulich, Capacity analysis and optimization of OFDM with distortionless PAPR reduction. Eur. Trans. Telecommun. 19, 781-790 (2008)

29. S Woo, H Yu, J Lee, C Lee, J Laskar, Effects of RF impairments in transmitte for the future beyond-3 G communications systems, in Proceedings of the 2006 IEEE International Symposium on Circuits and Systems (ISCAS), Island of Kos, Greece, 2006, pp. 5668-5671

30. PB Kenington, RF and Baseband Techniques for Software Defined Radio, 1st edn. (Norwood, MA, Artech House, 2005), p. 8

31. Y Rong, Y Xiang, Multiuser multi-hop MIMO relay systems with correlated fading channels. IEEE Trans. Wirel. Commun. 10(9), 2835-2840 (2011)

32. Q Li, KH Li, KC Teh, Achieving optimal diversity-multiplexing tradeoff for full-duplex MIMO multihop relay networks. IEEE Trans. Inf. Theory 57(1), 303-316 (2011)

\section{doi:10.1186/1687-1499-2013-32}

Cite this article as: Ahmad et al.: Spectral broadening effects of highpower amplifiers in MIMO-OFDM relaying channels. EURASIP Journal on Wireless Communications and Networking 2013 2013:32.

\section{Submit your manuscript to a SpringerOpen ${ }^{\odot}$ journal and benefit from:}

- Convenient online submission

- Rigorous peer review

- Immediate publication on acceptance

- Open access: articles freely available online

- High visibility within the field

- Retaining the copyright to your article

Submit your next manuscript at $\gg$ springeropen.com 